\section{Avaliação de fatores clínicos, laboratoriais e ultrassonográficos preditores de malignidade em nódulos tiroidianos}

\author{
Evaluation of clinical, laboratorial and ultrasonographic \\ predicting factors of malignancy in thyroid nodules
}

Ana Luiza Silva Rio ${ }^{1,2}$, Rosa Paula Mello Biscolla ${ }^{1,2,3}$, Danielle Macellaro Andreoni ${ }^{1,2}$, Cléber Pinto Camacho ${ }^{1,2}$, Cláudia Cristina Doimo Nakabashi 1,2, Maria da Conceição de Oliveira Carneiro Mamone ${ }^{1,2}$, Elza Setsuku Ikejiri' ${ }^{12}$, Luiza K. Matsumura', Jairo Tabacow Hidal',2, Rui Monteiro de Barros Maciel',2,3, Reinaldo Perrone Furlanetto'

\section{RESUMO}

Objetivo: Avaliar risco de malignidade de nódulos tiroidianos por meio de aspectos clínicos, laboratoriais, ultrassonográficos e citológicos. Pacientes e métodos: 741 nódulos de 407 pacientes. Resultados: A citologia foi benigna $(60,5 \%)$, indeterminada $(23,3 \%)$, maligna $(8,6 \%)$ ou não diagnóstica (7,6\%). A prevalência de câncer nas citologias indeterminadas foi $18,5 \%$ (16\% nas lesões foliculares, $44 \%$ nas suspeitas). 0 diagnóstico de malignidade foi $17,2 \%$ ( $n=70$ ). A frequência de câncer em mulheres $(15,2 \%)$ foi menor do que em homens (27,9\%). Houve uma relação inversa entre idade e risco de câncer. Não houve significância estatística na prevalência de câncer de acordo com número, tamanho dos nódulos ou níveis deTSH. Hipoecogenicidade e microcalcificações ao ultrassom foram fatores de risco. Conclusão: 0 risco de malignidade foi maior em homens, nódulos hipoecogênicos, com microcalcificações e inversamente relacionado à idade. O nível deTSH não foi um preditor independente de malignidade. Arq Bras Endocrinol Metab. 2011;55(1):29-37

Descritores

Nódulo de tiroide; sexo masculino; idade; ultrassonografia, punção de tiroide

\begin{abstract}
Objective: To evaluate the risk of malignancy in thyroid nodules through clinical, laboratory, ultrasonographic and cytological aspects. Patients and methods: 741 nodules of 407 patients. Results: The cytology was benign $(60,5 \%)$, indeterminate $(23,3 \%)$, malignant $(8,3 \%)$ or nondiagnostic $(7,6 \%)$. The prevalence of cancer in indeterminate citology was $18,5 \%$ (16\% in follicular lesions, $44 \%$ in suspicious). The diagnosis of malignancy was $17,2 \%(n=70)$. The frequency of cancer in women $(15,2 \%)$ was lower than in men $(27,9 \%)$. There was an inverse relation between age and cancer risk. There was no statistical significance in the prevalence of cancer according to number, size of nodules or TSH levels. Hypoechogenicity and microcalcifications on ultrasound were risk factors. Conclusion: The risk of malignancy was higher in men, hypoechoic nodules, with microcalcifications and was inversely related to age. The TSH level was not an independent factor predictive of malignancy. Arq Bras Endocrinol Metab. 2011;55(1):29-37
\end{abstract}

\section{Keywords}

Thyroid nodules; male sex; age; ultrasonography; fine needle aspiration biopsy
${ }^{1}$ Disciplina de Endocrinologia, Departamento de Medicina, Escola Paulista de Medicina, Universidade Federal de São Paulo (Unifesp/ EPM), São Paulo, SP, Brasil ${ }^{2}$ Centro de Doenças Tiroidianas, Instituto Israelita de Ensino e Pesquisa Albert Einstein (IIEPAE), São Paulo, SP, Brasil ${ }^{3}$ Fleury Medicina e Saúde, São Paulo, SP, Brasil

\section{Correspondência para: Ana Luiza Silva Rio Av. Almirante Alexandrino de Alencar, 1618, ap. 301 59015-350 - Natal, RN, Brasil anario01@hotmail.com}

Recebido em 30/Set/2010 Aceito em 13/Jan/2011

\section{INTRODUÇÃO}

$\mathrm{D}$ e acordo com estudos epidemiológicos, nódulos tiroidianos (NT) palpáveis ocorrem em 3\% a $8 \%$ da população (1-2). Estudos de autópsia demonstram que metade da população apresenta NT (3). O diagnóstico de nódulos não palpáveis aumentou em decorrência do 
uso de exames ultrassonográficos de alta resolução, podendo ser encontrados em $19 \%$ a $67 \%$ da população (4) com ocorrência maior em idosos, indivíduos do sexo feminino e áreas de baixo consumo de iodo $(4,5)$. Em consequência do maior uso de exames de imagem, há também um aumento da prevalência de microcarcinoma de tiroide, definido pela Organização Mundial da Saúde (OMS) como câncer de tiroide $\leq 10 \mathrm{~mm}$ (6). A importância de se avaliar um NT encontra-se, principalmente, na necessidade de se excluir câncer de tiroide que ocorre em 5\%-15\% dos casos $(2,7)$.

$\mathrm{Na}$ literatura, parâmetros clínicos de maior suspeita de malignidade em NT incluem: idade menor de 20 anos ou maior de 70 anos, sexo masculino, história familiar de câncer de tiroide, exposição à radiação ionizante, sintomas compressivos, nódulos de crescimento rápido, diâmetro maior que $4 \mathrm{~cm}$, nódulos endurecidos, aderidos a estruturas adjacentes, presença de paralisia de cordas vocais e linfadenopatia regional $(4,5,8)$.

$\mathrm{O}$ risco de malignidade em nódulos múltiplos é controverso na literatura. Alguns autores acreditam que a taxa de malignidade em nódulos solitários é maior do que em bócios multinodulares (BMN) (9-10), no entanto, recentemente, alguns trabalhos mostraram taxas semelhantes nos dois grupos $(2,7,11,12)$.

Em relação aos exames laboratoriais, alguns estudos sugerem que o nível de hormônio tiroestimulante (TSH) na apresentação clínica pode ser um preditor independente de malignidade $(9,13)$.

Características ultrassonográficas podem também auxiliar na avaliação do risco de malignidade em NT. São considerados nódulos suspeitos aqueles hipoecogềnicos, sólidos ou mistos com componente sólido, mal delimitados, sem halo e com aumento do fluxo nodular central visto pelo Doppler (14-17). No entanto, não é possível diferenciar com certeza lesões malignas de benignas somente pela ultrassonografia (US).

A punção aspirativa por agulha fina (PAAF) com estudo citológico do material obtido permanece até o momento como a técnica "gold-standart" na avaliação de NT $(2,18)$. Vários estudos demonstraram que a PAAF guiada por US em comparação com a PAAF guiada pela palpação aumenta a sensibilidade e diminui o número de aspirados não diagnósticos $(9,19)$.

Em vista do exposto, a proposta do estudo foi avaliar fatores de risco para câncer de tiroide já bem estabelecidos como idade, sexo, características ultrassonográficas, assim como fatores controversos como TSH, na tentativa de auxiliar na decisão de quais NT devem realmente ser encaminhados para tratamento cirúrgico.

\section{PACIENTES E MÉTODOS}

\section{Pacientes}

Este estudo foi aprovado pelo Comitê de Ética em Pesquisa do Hospital São Paulo, Universidade Federal de São Paulo (Unifesp).

Foram analisados dados referentes às características de $741 \mathrm{NT}$ de 407 pacientes (257 prospectivos e 150 retrospectivos) atendidos no período de outubro de 2006 a agosto de 2008 referenciados ao Centro de Doenças Tiroidianas, uma parceria entre a Unifesp e o Instituto Israelita de Ensino e Pesquisa Albert Einstein (IIEPAE). O critério para encaminhamento desses pacientes foi a presença de NT pelo exame físico, nódulos diagnosticados incidentalmente por exames de imagem (US, tomografia computadorizada, ressonância nuclear magnética), além de NT previamente conhecidos e que eram encaminhados para reavaliação. Não foram incluídos nódulos tóxicos.

Todos os pacientes foram submetidos à anamnese detalhada levando-se em consideração a faixa etária, a presença de sintomas de disfunção tiroidiana, a exposição à radiação ionizante e o exame físico, incluindo palpação criteriosa da região cervical. Além do atendimento clínico, os pacientes realizaram US de tiroide e dosagem de TSH. Foram analisadas características ultrassonográficas, tais como número de nódulos, tamanho, presença de calcificações, presença de halo e delimitação do nódulo para correlacioná-las com maior risco de malignidade. A realização de US foi feita por um único profissional.

Pacientes com níveis de TSH dentro dos limites da normalidade foram submetidos à PAAF de nódulos maiores ou iguais a $1 \mathrm{~cm} \mathrm{e} /$ ou com características suspeitas ao US. Aqueles com níveis de TSH abaixo ou acima do limite da normalidade foram avaliados quanto à presença de hipertiroidismo ou hipotiroidismo, respectivamente, e tratados antes de serem submetidos à PAAF no intuito de minimizar o risco de alterações no resultado da citologia aspirativa. Pacientes com diagnóstico de hipertiroidismo por bócio nodular tóxico não foram puncionados e foram excluídos deste estudo.

Os achados citológicos foram classificados em benignos, malignos, indeterminados (incluindo lesão folicular e lesões suspeitas) e não diagnósticos (incluindo material insuficiente e sangue) de acordo com a classificação da American Thyroid Association (2006) (2). A interpretação da citologia aspirativa foi feita sempre pelo mesmo profissional. Pacientes com PAAF benigna 
foram encaminhados para seguimento clínico. Nódulos com PAAF maligna, indeterminada ou insuficiente em mais de uma ocasião e nódulos volumosos com sintomas compressivos foram encaminhados para tiroidectomia. Dados do estudo anatomopatológico foram obtidos para análise dos resultados.

\section{Métodos}

A dosagem de TSH foi realizada pelo método de quimioluminescência (valores de referência: 0,35 a 5,5 $\mathrm{mcUI} / \mathrm{ml}$ com limite de detecção $0,01 \mathrm{mcUI} / \mathrm{ml}$ ).

Para a realização de US de tiroide foi utilizado o aparelho da marca ATL com Doppler colorido modelo HDI 3500 e com transdutor linear de 7,5-10 MGz.

O material obtido pela PAAF foi fixado e corado pela técnica Panótico.

\section{Análise estatística}

Após análise das características dos NT, realizou-se análise estatística dos dados para avaliação dos fatores associados com maior risco para malignidade.

Para a comparação de dados categóricos, o teste Qui-quadrado $\left(X^{2}\right)$ (sem a correção de Yates) foi utilizado obedecendo-se as restrições de Cochran e, quando estas estiveram presentes, foi realizado o teste exato de Fisher.

Diferenças entre médias de dados contínuos foram testadas utilizando-se os testes T de Student $(t)$ e Mann-Whitney. Como ambos mostraram resultados similares, somente os resultados dos testes paramétricos foram mostrados.

A regressão logística múltipla foi usada para investigar as relações individuais entre as variáveis independentes (aspectos clínicos e de US) e dependentes (presença de malignidade). Usamos o critério stepwise forward likelihood ratio (SFLR) para a seleção das variáveis independentes. Os resultados dessas análises foram calculados pelo teste de Wald (Wald $X^{2}$ ). As suposições dessas análises foram verificadas. Os resultados estão apresentados em Razão das Chances (Odds Ratio - OR).

A probabilidade $(p)$ menor que 0,05 foi considerada para indicar significância estatística; todos os testes foram bicaudados. Noventa e cinco por cento de intervalo de confiança (IC) foram calculados em relação às diferenças entre médias e OR. Toda a análise foi calculada segundo o programa estatístico SPSS (Statistical Package for the Social Science) 13.1 para Windows.

\section{RESULTADOS}

\section{Dados descritivos}

Foram avaliadas as características de 741 NT de 407 pacientes. Destes, $364(89 \%)$ eram mulheres $(8,5$ \% : 1 $\widehat{)}$ ). A idade média dos pacientes foi de 49,9 $\pm 14,7$ anos (variação: 11 a 84 anos). Nenhum paciente referia história de exposição à radiação ionizante. $\mathrm{O}$ nódulo encaminhado para avaliação foi percebido pelo próprio paciente $(36 \%)$, diagnosticado por US de tiroide $(32 \%)$ ou por um médico $(28 \%)$.

Foram avaliados 206 (51\%) pacientes com bócio uninodular e $200(49 \%)$ pacientes com BMN. Destes, $109(54 \%)$ apresentaram dois nódulos, 59 (29\%), três nódulos, 21 (10\%), quatro nódulos, $10(5 \%)$, cinco nódulos e um paciente apresentou seis nódulos. Um paciente encaminhado com suspeita clínica de NT apresentava bócio difuso ao US (Figura 1).

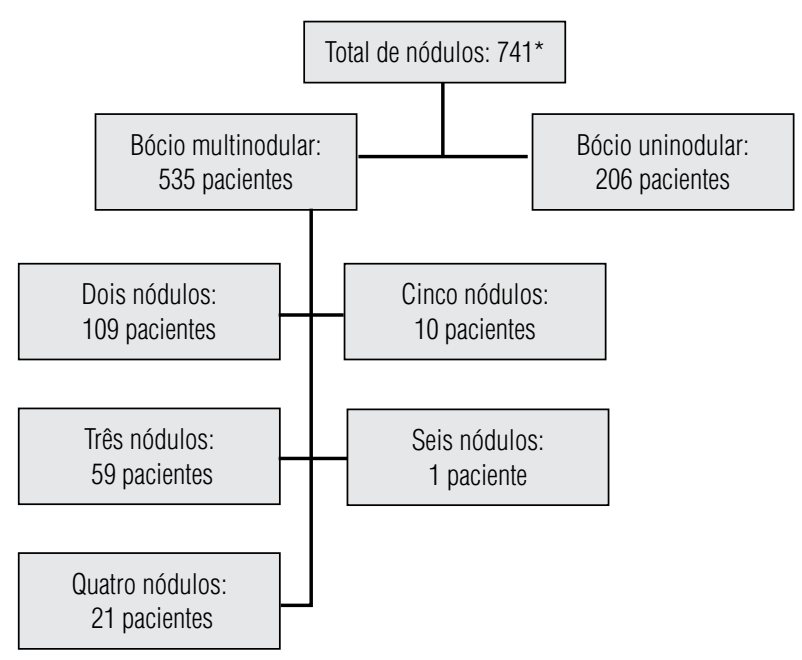

* Uma paciente encaminhada para avaliação de nódulo apresentava na realidade bócio com aspecto pseudonodular e PAAF compatível com linfoma.

Figura 1. Distribuição dos nódulos tiroidianos de acordo com o número de pacientes.

Do total de 741 NT confirmados ao US, 206 eram menores que $1 \mathrm{~cm}$, dos quais $59(28,6 \%)$ foram puncionados. Do total de 535 NT maiores ou iguais a $1 \mathrm{~cm}$, $453(84,7 \%)$ foram puncionados.

\section{Dados citológicos e histopatológicos}

O resultado citológico final dos NT puncionados foi benigno $(60,5 \%)$, indeterminado $(23,3 \%)$, maligno $(8,6 \%)$ ou não diagnóstico $(7,6 \%)$ (Figura 2$)$. Dos nódulos menores que $1 \mathrm{~cm}$ submetidos à PAAF, 25,4\% 
(15 de 59 NT) foram não diagnósticos, enquanto $5,3 \%$ (24 de $453 \mathrm{NT}$ ) dos nódulos maiores ou iguais a $\mathrm{l} \mathrm{cm}$ tiveram esse diagnóstico citológico. Portanto, o número de PAAF com resultado não diagnóstico em nódulos menores que $1 \mathrm{~cm}$ foi cinco vezes maior do que em nódulos maiores ou iguais a $\mathrm{l} \mathrm{cm}$.

Um total de 164 pacientes foi submetido à cirurgia. Dos 40 pacientes com PAAF sugestivas de carcinoma papilífero submetidos à cirurgia, dois pacientes tiveram resultados falso-positivos (bócio adenomatoso). Do total de 64 pacientes com PAAF sugestivas de benignidade encaminhados para cirurgia, dois apresentaram resultados falso-negativos (carcinoma papilífero), um deles associado à tiroidite linfocítica. A acurácia da PAAF quando o resultado final era compatível com malignidade foi de $96 \%$, a sensibilidade, $95 \%$, a especificidade, $97 \%$, o valor preditivo positivo, $95 \%$, e o valor preditivo negativo foi $97 \%$.

Dos 119 nódulos com PAAF indeterminadas, 114 foram encaminhados para tratamento cirúrgico e cinco tiveram contraindicação para cirurgia. Dos 103 nódulos operados, foram encontradas 94 lesões foliculares e nove suspeitas. A prevalência de câncer em NT com PAAF indeterminada foi de $18,5 \%$ (16\% nas lesões foliculares e de $44 \%$ nas lesões suspeitas). Nos NT com diagnóstico na PAAF de lesão folicular, $84 \%$ apresentaram resultado final benigno, $10,7 \%$, carcinoma papilífero, $4,3 \%$, carcinoma folicular e $1 \%$ apresentou carcinoma medular. Já os NT com PAAF suspeita apresentaram $55,6 \%$ de benignidade, $33,3 \%$ de carcinoma papilífero e $11,1 \%$ de carcinoma folicular (Figura 3 ).

A prevalência final de malignidade em pacientes com NT foi de $17,2 \%$ (70/407), sendo o carcinoma papilífero o tipo histológico mais frequente de câncer de tiroide $(87,2 \%)$ (Tabela 1$)$.

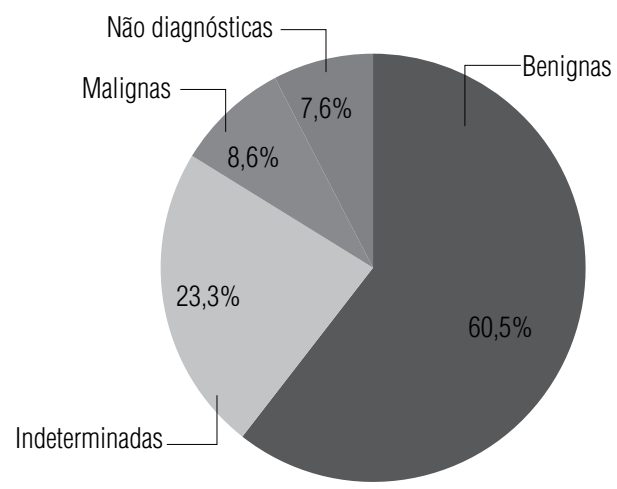

Figura 2. Resultado citológico de 513 nódulos avaliados pela punção aspirativa.

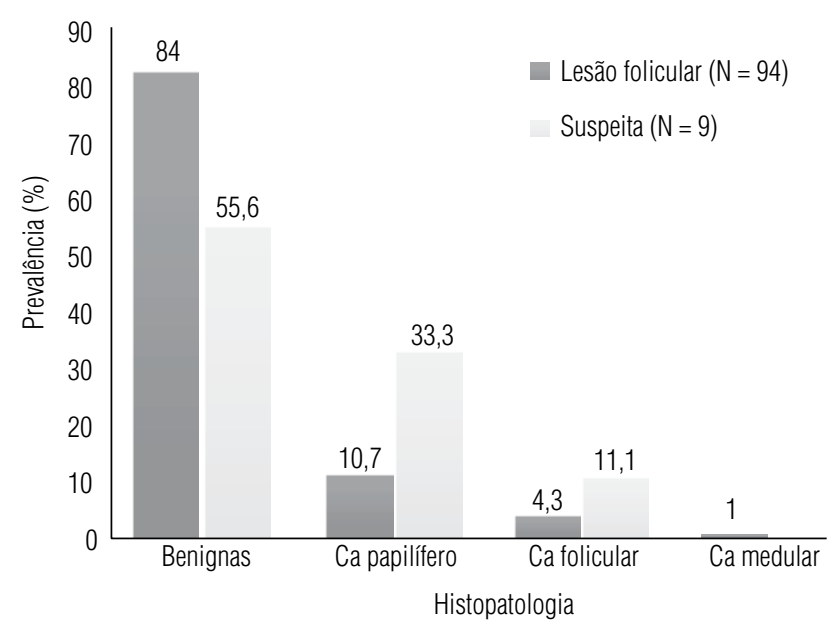

Figura 3. Resultado histopatológico de 103 nódulos com punção aspirativa indeterminada.

Tabela 1. Distribuição dos tipos de câncer de tiroide diagnosticados

\begin{tabular}{lc}
\hline Tipos de câncer de tiroide & N (\%) \\
\hline Carcinoma papilifero & $61(87,2)$ \\
Carcinoma folicular & $05(7,2)$ \\
Carcinoma medular & $01(1,4)$ \\
Linfoma & $01(1,4)$ \\
Indiferenciado & $01(1,4)$ \\
Metástase de câncer de mama & $01(1,4)$ \\
Total & $70(100)$ \\
\hline
\end{tabular}

\section{Características clínicas}

A frequência de câncer de tiroide em mulheres com nódulos tiroidianos foi significativamente menor do que a de homens: $15,2 \%$ em mulheres e $27,9 \%$ homens $(\mathrm{p}=$ $0,034)$. Não houve diferença estatística na prevalência de câncer de tiroide entre pacientes com nódulo único $(15,6 \%)$ ou com BMN $(17,5 \%)(\mathrm{p}=0,609)$.

A prevalência de microcarcinoma em pacientes com câncer de tiroide foi de 25,7\% (18/70), sendo que em todos os casos tratava-se de microcarcinoma papilífero e em $94,4 \%$ dos casos estava presente em mulheres.

Em nove casos $(50 \%)$ o diagnóstico de microcarcinoma foi incidental e em três destes os nódulos não haviam sido visualizados previamente no US. Os outros $50 \%$ foram puncionados anteriormente à cirurgia por apresentarem características ultrassonográficas suspeitas $(\mathrm{n}=4)$ ou diâmetro maior que $\mathrm{l} \mathrm{cm}$ ao US $(\mathrm{n}=5)$. O tamanho médio dos microcarcinomas no histopatológico foi de $6,6 \mathrm{~mm}$ (variação de 3 a $10 \mathrm{~mm}$ ) com $77,8 \%$ dos nódulos maiores que $5 \mathrm{~mm}$. 


\section{Características laboratoriais}

Foram analisados os valores de TSH de pacientes submetidos à cirurgia. Para essa análise, foram incluídos pacientes com carcinoma diferenciado de tiroide (CDT) com valores de TSH dentro da normalidade e sem reposição de LT4.

A média de TSH dos pacientes com nódulos benig$\operatorname{nos}(\mathrm{n}=77)$ não foi estatisticamente diferente da média dos pacientes com nódulos malignos $(\mathrm{n}=60)(1,57 \pm$ $1,06 \mathrm{mcUI} / \mathrm{ml}$ vs. $1,77 \pm 1,09 \mathrm{mcUI} / \mathrm{ml}, \mathrm{p}=0,291)$.

A fim de se excluir uma possível interferência nos valores de TSH, a análise foi feita excluindo os pacientes com tiroidite linfocítica. A média de TSH dos pacientes com nódulos benignos ( $\mathrm{n}=55$ ) também não foi estatisticamente diferente da média dos pacientes com nódulos malignos $(\mathrm{n}=48)(1,44 \pm 1,04 \mathrm{mcUI} / \mathrm{ml} v \mathrm{~s}$. $1,71 \pm 1,11 \mathrm{mcUI} / \mathrm{ml}, \mathrm{p}=0,203)$.

Foi feita a distribuição dos pacientes com câncer para avaliar o aumento do risco de malignidade de acordo com os níveis de TSH ao diagnóstico. Essa análise incluiu nódulos com histopatologia de benignidade e CDT. Os dados de concentração sérica de TSH foram subdivididos a partir de valores determinados pela literatura $(13,20-22)$ (Figura 4$)$.

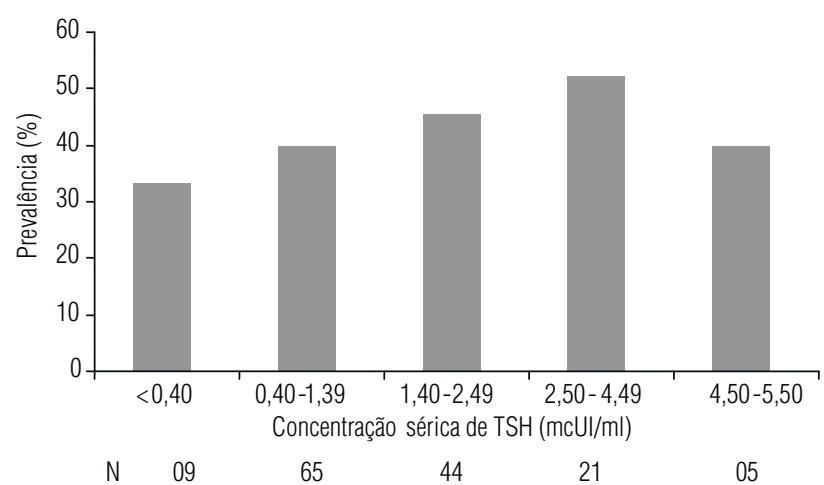

Figura 4. Prevalência de malignidade em 144 pacientes com nódulo de tiroide de acordo com a concentração sérica de TSH ao diagnóstico.

Não houve diferença significante entre a prevalência de malignidade e a concentração sérica de TSH (teste exato de Fisher; $n=144 ; \mathrm{p}=0,291$ ).

Não houve diferença significativa na prevalência de CDT em pacientes com ou sem tiroidite linfocítica confirmada pela histopatologia (benignos $\mathrm{n}=97$ e malig$\operatorname{nos} \mathrm{n}=66)(31,7 \%$ vs. $43,9 \%, \mathrm{p}=0,185)$.

\section{Relação entre achados de US de CDT nos NT}

Para essa análise foram incluídos dados de pacientes com diagnóstico histopatológico de benignidade, CDT e os com PAAF benignas não submetidos à cirurgia. Os pacientes com neoplasia indiferenciada, linfoma, câncer de mama e incidentalomas (micronódulos malignos encontrados na histopatologia não identificados previamente ao US) foram excluídos.

Não houve diferença significativa em relação ao tamanho dos nódulos benignos e malignos $(22,6 \pm 14,6$ $\mathrm{mm}$ vs. $24,1 \pm 19,8 \mathrm{~mm}, \mathrm{p}=0,431)$.

A análise das características ultrassonográficas dos NT evidenciou que as lesões malignas em relação às benignas eram mais frequentemente hipoecogênicas $(\mathrm{p}<0,001)$, sólidas $(\mathrm{p}=0,001)$, mal delimitadas $(\mathrm{p}=$ $0,036)$, sem halo $(\mathrm{p}<0,001)$ e com microcalcificações $(\mathrm{p}<0,001)$ (Tabela 2$)$.

Com a finalidade de identificar a combinação de parâmetros de US (variáveis independentes) mais relevantes no diagnóstico de malignidade (variável dependente) por regressão logística, incluímos as variáveis independentes que foram significantes na análise univariada. Usamos o critério SFLR para a seleção das variáveis independentes (Tabela 3 ).

O modelo final incluiu as variáveis microcalcificações e hipoecogenicidade. A presença de hipoecogenicidade aumenta em 5,18 vezes a chance de malignidade, enquanto a presença de microcalcificação aumenta em 15,57 vezes essa chance.

Tabela 2. Frequência das características ultrassonográficas de nódulos malignos e benignos

\begin{tabular}{lcc}
\hline & Malignos (\%) & Benignos (\%) \\
\hline Ecogenicidade $(\mathrm{n}=466)$ & & \\
Hiperecogênico & 2,8 & 3,3 \\
Hipoecogênico & 84,5 & $49,4(\mathrm{p}<0,001)$ \\
Isoecogênico & 12,7 & 47,3 \\
Composição ( $\mathrm{n}=542)$ & & \\
Cístico & 0 & 5,8 \\
Sólido & 83,8 & $64,7(\mathrm{p}=0,001)$ \\
Misto & 4,1 & 7,9 \\
Predominantemente cístico & 6,8 & 4,1 \\
Predominantemente sólido & 5,4 & 17,5 \\
Delimitação ( $\mathrm{n}=261)$ & & \\
Mal delimitado & 84,2 & $67,2(\mathrm{p}=0,036)$ \\
Bem delimitado & 15,8 & 32,3 \\
Halo ( $\mathrm{n}=408)$ & \\
Presente & & 53,6 \\
Ausente & 28,1 & $1,9(\mathrm{p}<0,001)$ \\
Microcalcificações ( $\mathrm{n}=554)$ & 71,9 & 98,1 \\
Presente & & \\
Ausente & 73,1 & $\mathrm{n}<0,001)$ \\
\hline
\end{tabular}




\section{Relação entre CDT e os achados clínicos nos pacientes com NT}

Para essa análise também incluímos os pacientes com diagnóstico histopatológico de CDT, benignidade e os pacientes com PAAF benignas não submetidos à cirurgia. Os pacientes com neoplasia indiferenciada, linfoma, câncer de mama e incidentalomas (micronódulos malignos encontrados na histopatologia não identificados previamente ao US) foram excluídos.

Entre os pacientes com NT, a proporção de CDT foi maior em homens do que em mulheres $(38,2 \% v s$. $17,4 \%, \mathrm{n}=332, \mathrm{p}=0,004)$; em nódulos com hipoecogenicidade $(28,2 \%$ vs. $6,4 \%, \mathrm{n}=297, \mathrm{p}<0,001)$ e naqueles com microcalcificação $(65,5 \%$ vs. $15,2 \%, \mathrm{n}=$ $332, \mathrm{p}<0,001)$.

Não houve diferença significativa em relação ao número de nódulos entre os pacientes com e sem CDT $(1,98 \pm 1,26 v s .1,72 \pm 0,95, \mathrm{p}=0,063)$ e em relação aos valores de TSH $(1,80 \pm 1,18 \mathrm{mcUI} / \mathrm{ml}$ vs. $1,71 \pm$ $1,19 \mathrm{mcUI} / \mathrm{ml}, \mathrm{p}=0,615)$. Porém, os pacientes com CDT apresentavam idade menor do que os sem CDT $(44,5 \pm 15,3$ anos vs. 50,1 $\pm 14,2$ anos, $\mathrm{p}=0,006)$.

Foi feita a análise por regressão logística para identificar a combinação de parâmetros clínicos e de US (variáveis independentes) mais relevantes no diagnóstico de malignidade (variável dependente). Para isso, incluímos as variáveis independentes citadas anteriormente que foram significantes na análise univariada. Foi utilizado o critério SFLR para a seleção das variáveis independentes (Tabela 4).
O modelo final incluiu as variáveis idade, presença de microcalcificações e hipoecogenicidade. A presença de pelo menos um nódulo hipoecogênico aumenta em 4,90 vezes o risco de malignidade, enquanto a presença de pelos menos um nódulo com microcalcificação aumenta em 8,28 vezes o risco de malignidade. Por outro lado, para cada ano de vida o risco de malignidade se reduz em 0,97 vez.

\section{DISCUSSÃO}

A proposta do presente estudo foi avaliar fatores de risco para câncer de tiroide na tentativa de auxiliar na decisão de quais NT devem realmente ser encaminhados para tratamento cirúrgico. Em nossos pacientes com NT, a prevalência de câncer foi de $17,2 \%$, aparentemente maior do que a observada em outros estudos (5\%-15\%) $(2,7,23)$, provavelmente por tratar-se de um serviço de referência em que alguns pacientes encaminhados foram previamente avaliados por endocrinologistas e apresentavam uma maior suspeita para malignidade.

Uma revisão de Hegedüs (4) mostrou que a frequência final dos resultados citológicos da PAAF varia de $53 \%$ a $90 \%$ para nódulos benignos, $5 \%$ a $23 \%$ para indeterminados, $1 \%$ a $10 \%$ para malignos e $15 \%$ a $20 \%$ para não diagnósticos. Em nosso estudo, observamos um menor número de PAAF não diagnósticas $(7,6 \%)$, provavelmente pela grande experiência do puncionador e do citologista que participaram do estudo. Banks e cols. (24), avaliando 639 pacientes com lesões indeter-

Tabela 3. Resultados das análises de regressão logística múltipla (SFLR) para o diagnóstico de malignidade, a partir da média dos parâmetros de ultrassonografia, como variáveis independentes

\begin{tabular}{lcccccc}
\hline Variável & $\boldsymbol{\beta}$ & Erro-padrão & Wald X & OR & 95\% IC (OR) & p \\
\hline Constante & $-3,49$ & 0,74 & - & - & - & - \\
Hipoecogenicidade & 1,64 & 0,80 & 4,25 & 5,18 & 1,08 a 24,74 & 0,039 \\
Microcalcificações & 2,75 & 0,79 & 11,93 & 15,57 & 3,28 a 73,94 & 0,001 \\
Demais parâmetros & \multicolumn{7}{c}{ Não selecionados } \\
\hline
\end{tabular}

Tabela 4. Resultados das análises por regressão logística múltipla (SFLR) para o diagnóstico de malignidade, a partir da média dos parâmetros clínicos e de ultrassonografia, como variáveis independentes

\begin{tabular}{lcccccc}
\hline Variável & $\beta$ & Erro-padrão & Wald X & OR & 95\% IC (OR) & p \\
\hline Constante & $-1,29$ & 0,63 & - & - & - & - \\
Hipoecogenicidade & 1,59 & 0,44 & 12,92 & 4,90 & 2,06 a 11,66 & $<0,001$ \\
Microcalcificações & 2,11 & 0,47 & 20,56 & 8,28 & 3,32 a $20,65<$ & $<0,001$ \\
Idade & $-0,03$ & 0,01 & 8,33 & 0,97 & 0,95 a 0,99 & 0,004 \\
Sexo & \multicolumn{7}{c}{ Não selecionada } \\
\hline
\end{tabular}


minadas na PAAF, encontraram $30 \%$ e $62 \%$ de malignidade em lesões foliculares e suspeitas de carcinoma, respectivamente. Outros autores mostraram uma prevalência de malignidade variando entre $12 \%$ e $40 \%$ em lesões indeterminadas pela PAAF (25-26). Em nosso estudo, os resultados foram semelhantes: a prevalência de câncer em nódulos com PAAF indeterminada foi de $18,5 \%$, sendo $16 \%$ nas lesões foliculares e $44 \%$ nas lesões suspeitas.

Comparando os nossos resultados com a nova classificação das PAAF pelo Sistema Bethesda (2009) (27), podemos observar que nossas lesões foliculares apresentam risco de malignidade (16\%) semelhante ao do grupo de lesões foliculares de significado indeterminado da classificação atual ( $5 \%$ a $15 \%)$. Já os nossos pacientes com lesões suspeitas (risco de $44 \%$ de malignidade) provavelmente englobam pacientes que seriam atualmente classificados nos grupos de neoplasia folicular (risco de $20 \%$ a $30 \%$ ) e de suspeitos para malignidade (risco de $50 \%$ a $75 \%(27)$.

O tipo histopatológico mais frequente de câncer de tiroide é o carcinoma papilífero $(80 \%$ a $87 \%)(7,13,18)$ e observamos uma ocorrência em nosso estudo de $87,2 \%$. Entre os pacientes com câncer de tiroide, $25,7 \%$ apresentavam microcarcinoma de tiroide, todos do tipo papilífero. O microcarcinoma de tiroide em $65 \%$ a $99 \%$ é do tipo papilífero (28) e sua prevalência tem aumentado (29); Roti e cols. (28) concluíram que microcarcinoma papilífero está presente em um quarto dos casos de malignidade de tiroide. O principal fator associado ao aumento do diagnóstico de microcarcinoma papilífero é o uso do US de tiroide. A recomendação das diversas sociedades é que nódulos menores que $1 \mathrm{~cm}$ sejam puncionados somente na presença de características suspeitas, linfadenopatia, história de irradiação cervical ou história familiar de câncer de tiroide em parentes de primeiro grau $(17,18,30)$. Em nosso estudo, os microcarcinomas papilíferos que foram diagnosticados pela PAAF apresentavam ao menos duas características ultrassonográficas suspeitas, tais como hipoecogenicidade, ausência de halo ou presença de microcalcificações.

Em relação aos dados clínicos, os indivíduos do sexo masculino apresentaram maior risco de malignidade do que as mulheres $(27,9 \%$ e $15,2 \%$, respectivamente $)$ conforme observado também por outros autores $(13,23)$.

Por meio da análise de regressão múltipla observamos uma relação inversa entre idade e risco de malignidade. Rago e cols. (31) recentemente demonstraram resultados semelhantes na avaliação do risco para carci- noma papilífero de tiroide. Alguns estudos, no entanto, demonstraram uma maior ocorrência de câncer de tiroide em pacientes com idade abaixo de 30 anos e acima de 70 anos $(2,9,13,23,24)$. Em nosso estudo, porém, não houve um aumento de risco de câncer com o aumento da faixa etária. Alguns dados recentes citados em uma revisão de Alexander (23) são contraditórios em relação ao maior risco de câncer em indivíduos mais idosos, permanecendo incerto se realmente esse risco existe, sendo prudente assumir que, considerando a idade do paciente, indivíduos mais jovens apresentam maior risco $(11,23)$.

Boelaert e cols. (9) observaram um risco maior de malignidade em nódulo único comparado com pacientes com bócio multinodular e bócio difuso (9); no entanto, observamos risco semelhante de malignidade em nódulo único e em bócio multinodular, de acordo com a maioria dos trabalhos recentes $(7,12,14,18)$.

A relação do TSH com CDT não está bem estabelecida. Já se demonstrou que no CDT não ocorre mutação no receptor de TSH associada com aumento de transdução (32); outros fatores como IGF-1 são importantes para crescimento tumoral e existe uma relação inversa entre nível de RNAm do receptor de TSH e agressividade do tumor (33-34). Por outro lado, há evidência clara do aumento de sobrevida em pacientes com câncer mantidos com TSH suprimido em uso de LT4 (35). Por essa razão, alguns autores avaliaram a concentração de TSH de pacientes com CDT e observaram maior risco de malignidade com o aumento dos seus níveis $(9,13)$. Em um desses estudos, a média de TSH no grupo de pacientes com câncer de tiroide foi mais alta (13). Nossos resultados não evidenciaram um maior risco de CDT com o aumento do TSH e não houve diferença entre as médias de TSH dos pacientes com ou sem câncer de tiroide.

Outros fatores de risco controversos avaliados em estudos recentes, como fatores de risco para câncer de tiroide, além do TSH, são: os níveis de leptina que se encontram elevados e diminuem após a tiroidectomia (36); o índice de massa corporal aumentado (37) e a presença de resistência insulínica, que tem se mostrado também como importante fator de risco para outros carcinomas não tiroidianos (38).

Ao analisarmos as características ultrassonográficas dos NT de forma univariada, observamos que a presença de hipoecogenicidade, ausência de halo, presença de microcalcificações e lesões mal delimitadas estavam presentes de forma significativa em NT malignos. Não 
houve diferença significante entre o tamanho dos nódulos benignos e malignos. A análise multivariada demonstrou que NT hipoecogênico aumenta em 4,9 vezes a chance de malignidade, enquanto a presença de pelos menos um nódulo com microcalcificação aumenta em 8,2 vezes a chance de malignidade. Frates e cols. (7) observaram que a composição do nódulo (quanto maior o componente sólido maior o risco), a hipoecogenicidade e a presença de calcificações foram fatores de risco para malignidade, enquanto a presença de halo e o tamanho do nódulo não foram de significado prognóstico. Papini e cols. (14) observaram que a presença de fluxo vascular intranodular avaliado pelo Doppler estava mais relacionado com malignidade assim como a presença de microcalcificações e lesões irregulares; a hipoecogenicidade e o tamanho do nódulo, no entanto, não foram fatores de risco.

Avaliamos a histopatologia para confirmar a presença ou não de tiroidite linfocitária crônica e não observamos associação com CDT. Para Boelaert e cols. (9), a presença de anticorpos antitiroidianos não foi considerada um fator de risco independente para malignidade. No entanto, em pacientes com tiroidite de Hashimoto e nódulos bem definidos a recomendação é que seja realizada a PAAF tanto para afastar a presença de CDT quanto de linfoma, embora este seja raro (17). Nosso paciente com diagnóstico de linfoma apresentava tiroidite de Hashimoto prévia ao diagnóstico de malignidade; a maior ocorrência de linfoma em pacientes com doença linfocitária crônica associada está bem estabelecida na literatura (39).

Concluímos que o risco de malignidade foi maior no sexo masculino, em nódulos hipoecogênicos e com microcalcificações vistos ao US. Houve uma relação inversa entre idade e risco de malignidade. O número de nódulos, o nível de TSH e a presença de tiroidite linfocítica crônica não foram fatores de risco para câncer de tiroide.

Agradecimentos: ao apoio da Coordenação de Aperfeiçoamento de Pessoal de Nível Superior (Capes) por meio de bolsa de Mestrado.

Declaração: os autores declaram não haver conflitos de interesse científico neste estudo.

\section{REFERÊNCIAS}

1. Wiest PW, Hartshome MF, Inskip PD, Crooks LA, Vela BS, Telepak RJ, et al. Thyroid palpation versus high-resolution thyroid ultrasonography in the detection of nodules. J Ultrasound Med. 1998;17(8):487-96.

2. Cooper DS, Doherty GM, Haugen BR, Kloos RT, Lee SL, Mandel $\mathrm{SJ}$, et al. Management guidelines for patients with thyroid nodules and differentiated thyroid cancer. Thyroid. 2006;16(2):1-24.

3. Tan GH, Gharib H. Thyroid incidentalomas: management approaches to nonpalpable nodules discovered incidentally on thyroid imaging. Ann Intern Med. 1997;126(3):226-31.

4. Hegedüs L. The thyroid nodule. N Eng J Med. 2004;351(17):1764-71.

5. Hegedüs L, Bonnema SJ, Bennedbaek FN. Management of simple nodular goiter: current status and future perspectives. Endocr Ver. 2003;24(1):102-32.

6. Lloyd R, De Lellis R, Heitz P, Eng C. World Health Organization: pathologic and genetics of tumors of endocrine organs. Lyon, França: IARC Press International Agency for Research on Cancer; 2004.

7. Frates $M C$, Benson $C B$, Doubilet $P M$, Kunreuther $E$, Contreras $M$, Cibas ES, et al. Prevalence and distribution of carcinoma in patients with solitary and multiple thyroid nodules on sonography. J Clin Endocrinol Metab. 2006;91(9):3411-7.

8. Shibata Y, Yamashita S, Masyakin VB, Panasyuk GD, Nagataki S. 15 years after Chernobyl: new evidence of thyroid cancer. Lancet. 2001;358(9297):1965-6.

9. Boelaert K, Horacek J, Holder RL, Watkinson JC, Sheppard MC, Franklyn JA. Serum thyrotropin concentration as a novel predictor of malignancy in thyroid nodules investigated by fine-needle aspiration. J Clin Endocrinol Metab. 2006;91(11):4295-301.

10. Mazzaferri EL. Management of a solitary thyroid nodule. N Engl J Med. 1993;328(8):553-9.

11. Belfiore A, La Rosa GL, La Porta GA, Giuffrida D, Milazzo G, Lupo $L$, et al. Cancer risk in patients with cold thyroid nodules: relevance of iodine intake, sex, age and multinodularity. Am J Med. 1992;93(4):363-9.

12. Marqusee $E$, Benson $C B$, Frates MC, Doubilet PM, Larsen PR, Cibas $E S$, et al. Usefulness of ultrasonography in the management of nodular thyroid disease. Ann Intern Med. 2000;133(9):696-700.

13. Haymart MR, Repplinger DJ, Leverson GE, Elson DF, Sippel RS, Jaume JC, et al. Higher serum TSH level in thyroid nodule patients is associated with greater risks of differentiated thyroid cancer and advanced tumor stage. J Clin Endocrinol Metab. 2008;93(3):809-14.

14. Papini E, Guglielmi R, Bianchini A, Crescenzi A, Taccogna S, Nardi $F$, et al. Risk of malignancy in nonpalpable thyroid nodules: predictive value of ultrasound and color-Doppler features. J Clin Endocrinol Metab. 2002;87(5):1941-6.

15. Nam-Goong IS, Kim HY, Gong G, Lee HK, Hong SJ, Kim WB, et al. Ultrasonography-guided fine-needle aspiration of thyroid incidentaloma: correlation with pathological findings. Clin Endocrinol. 2004;60(1):21-8.

16. Rago T, Vitti $P$, Chiovato SM, Mazzeo S, DeLiperi A, Miccolo P, et al. Role of conventional ultrasonography and color flow-Doppler sonography in predicting malignancy in "cold" thyroid nodules. Eur J Endocrinol. 1998;138(1):41-6.

17. Maia AL, Ward LS, Carvalho GA, Graf H, Maciel RMB, Maciel LMZ, et al. Nódulos de tireóide e câncer diferenciado de tireóide: consenso brasileiro. Arq Bras Endocrinol Metab. 2007;51(5):867-93.

18. Pacini F, Schlumberger $M$, Dralle $H$, Elisei $R$, Smit JWA, Wiersinga W. European consensus for the management of patients with differentiated thyroid carcinoma of the follicular epithelium. Eur J Endocrinol. 2006;154(6):787-803.

19. Castro MR, Gharib H. Continuing controversies in the management of thyroid nodules. Ann Intern Med. 2005;142(11):926-31.

20. Kratzsch J, Fiedler GM, Leichtle A, Brügel M, Buchbinder S, Otto $L$, et al. New reference intervals for thyrotropin and thyroid hormones based on National Academy of Clinical Biochemistry 
criteria and regular ultrasonography of the thyroid. Clin Chem. 2005;51(8):1480-6.

21. Hollowell JG, Staehling NW, Flanders D, Hannon WH, Gunter EW, Spencer CA, et al. Serum TSH, T4 and thyroid antibodies in The United States population (1988 to 1994): National Health and Nutrition Examination Survey (NHANES III). J Clin Endocrinol Metab. 2002;87(2):489-99.

22. Surks MI, Ortiz E, Daniels GH, Sawin CT, Col NF, Cobin RH, et al. Subclinical thyroid disease: scientific review and guidelines for diagnosis and management. JAMA. 2004;291(2):228-38.

23. Alexander EK. Approach to the patient with a cytologically indeterminate thyroid nodule. J Clin Endocrinol Metab. 2008;93(11):4175-82.

24. Banks ND, Kowalski J, Tsai HL, Somervell H, Tufano R, Dackiw $\mathrm{APB}$, et al. A diagnostic predictor model for indeterminate or suspicious thyroid FNA samples. Thyroid. 2008;18(9):933-41.

25. Goldstein RE, Burkey B, Johnson JE. Implications of follicular neoplasms, atypia and lesions suspicious of malignancy diagnosed by fine-needle aspiration of thyroid nodules. Ann Surg. 2002;235(5):656-64.

26. Kelman AS, Leibowitz J, Burstein DE, Haber RS. Thyroid cytology and the risk of malignancy in thyroid nodules: importance of nuclear atypia in indeterminate specimens. Thyroid. 2001;11(3):271-7.

27. Layfield LJ, Cibas ES, Gharib H, Mandel SJ. Thyroid aspiration cytology. Current Status. Cancer J Clin. 2009;59:99-110.

28. Roti E, Uberti EC, Bondanelli M, Braverman LE. Thyroid papillary microcarcinoma: a descriptive meta-analysis study. Eur J Endocrinol. 2008;159(6):656-73.

29. Leenhardt L, Grosclaude P, Chérié-Challine L. Thyroid Cancer Committee. Increase incidence of thyroid carcinoma in France: a true epidemic of thyroid nodule managements effect? Thyroid. 2004;14(12):1056-106.
30. Cooper DS, Doherty GM, Haugen BR, Kloos RT, Lee SL, Mandel $\mathrm{SJ}$, et al. Revised American Thyroid Association management guidelines for patients with thyroid nodules and differentiated thyroid cancer. Thyroid. 2009;19(11):1167-214.

31. Rago T, Fiore E, Scutari M, Santini F, Di Coscio G, Romani R, et al. Cytology suspicious for or indicative of PTC is associated with younger age, male sex, and solitary versus multiple nodularity. Eur J Endocrinol. 2010;162:763-70.

32. Matsuo K, Friedman E, Gejman PV, Fagin JA. The thyrotropin receptor (TSH-R) is not an oncogene for thyroid tumors: structural studies of the TSH-R and the $\alpha$-subunit of Gs in human thyroid neoplasms. J Clin Endocrinol Metab. 1993;76(6):1446-51.

33. Derwahl M, Broecker M, Kraiem Z. Thyrotropin may not be the dominant growth factor in benign and malignant thyroid tumors. J Clin Endocrinol Metab. 1999;84(3):829-34.

34. Mazzaferri EL. Thyroid cancer and Graves disease: the controversy ten years later. Endocr Pract. 2000;6(2):221-5.

35. Jonklaas J, Sarlis NJ, Litofsky D, Ain KB, Bigos ST, Bierley JD, et al. Outcomes of patients with differentiated thyroid carcinoma following initial therapy. Thyroid. 2006;16(12):1229-42.

36. Akinci M, Kosova F, Cetin B, Aslan S, Ari Z, Cetin A. Leptin levels in thyroid cancer. Asian J Surg. 2009;32(4):216-23.

37. Paes JE, Hua K, Nagy R, Kloos RT, Jarioura D, Ringel MD. The relationship between body mass index and thyroid cancer pathology features and outcomes: a clinicopathological cohort study. J Clin Endocrinol Metab. 2010;95(9):4244-50.

38. Rezzónico JN, Rezzónico M, Pusiol E, Pitoia F, Niepomniszcze $\mathrm{H}$. Increases prevalence of insulin resistance in patients with differentiated thyroid carcinoma. Metab Syndr Relat Disord. 2009;7(4):375-80.

39. Pasieka JL. Hashimoto's disease and thyroid lymphoma: role of the surgeon. World J Surg. 2000;24(8):966-70. 